\title{
Los años de formación de Wilhelm Meister: Tiempo, narración y Bildung
}

Fecha de recepción: 19/5/2020.

Fecha de aceptación: 20/10/2020.

\section{Resumen}

Es un error no considerar la relación que tiene el Bildungsroman con el tiempo, en tanto que este presenta, en su estructura narrativa-temporal, una representación del tiempo distintiva, específica a este subgénero novelístico. Las elipsis temporales y la dimensión del olvido; el acto de enfatizar, de volver visible, cierto tiempo presente e ideal; y la figura del joven como individuo temporal, sujeto a un determinado período vital; son variables que expresan la especificidad y autoconciencia de la representación temporal del Bildungsroman, como se ve en Los años de aprendizaje de Wilhelm Meister de J. W. Goethe, novela que pone en contacto, de un modo paradigmático, la dimensión temporal y la formativa. A su vez, la temporalidad permite reflexionar acerca de la variación histórica del subgénero, en una línea que llega a su punto culminante en el siglo XX, con Thomas Mann y La montaña mágica.

Palabras clave: Bildungsroman; filosofía del tiempo; narratología; Aufklärung.

Wilhelm Meister's Apprenticeship Years: Time, Narration and Bildung

\begin{abstract}
It is an error to not consider the relation that the Bildungsroman has with time, as it presents, in its narrative-temporal structures, a distinctive representation of time, specific to this novelistic subgenre. Temporal ellipsis and forgetfulness; the act of emphasizing, of making visible an ideal type of present-time; and the figure of youth as a temporary individual, subject to a certain life period; are
\end{abstract}


variables that express the specificity and self-awareness of the temporal representation of the Bildungsroman, as seen on J. W. Goethe's Wilhelm Meisters's Apprenticeship, a novel that connects, in a paradigmatic manner, the temporal and the formative dimensions. In addition, temporality allows us to reflect on the historical variation of this subgenre, in a line that reaches its climax in the 20th century with Thomas Mann and his The Magic Mountain.

Keywords: Bildungsroman; philosophy of time; narratology; Aufklärung.

[L]as artes humanas conocidas son sucesos naturales que proceden de un modo abierto o secreto. De este tipo es la adivinación. Esta reconoce lo oculto en lo revelado, lo futuro en lo presente, lo vivo en lo muerto y distingue el sentido de lo carente de sentido.

J. W. Goethe, Los años itinerantes de Wilhelm Meister (1829)

\section{Tiempo y formación}

Ora por su relevancia histórico-cultural (en su contexto y posteriormente), ora por su mérito estético, la novela de Goethe, Los años de aprendizaje de Wilhelm Meister (1795/96), ha encontrado un lugar -aunque, lamentablemente, no siempre acompañada de su presencia concreta, textual- en teorías sobre la novela o la representación del tiempo en la narrativa. Aún más ubicua resulta al considerarse su rol modélico, cuando no fundador, del Bildungsroman alemán o europeo (Sammons, 1991). Así, aparece en una posición privilegiada en la Teoría de la novela de Lukács (2002), como un caso especial de cronotopo en Bajtín (2013) y, en conjunto con novelas de Jane Austen, como exponente "clásico" de Bildungsromane en Moretti (1985; 1987). Adicionalmente, en textos fundacionales sobre tiempo y narración, como los de Günther Müller (2014) o Käte Hamburger (1994), descubriremos algún análisis, ${ }^{1}$ alguna referencia más o menos extensa sobre el Wilhelm Meister, ya sea como una unidad autónoma o analizada junto a su continuación, Los años de viaje.

Ahora bien, por un lado, cuando se estudia su dimensión narrativa-temporal, como hace Müller, la novela goetheana parece ingresar solo por ser una novela, sin ser considerada detenidamente su vinculación con las nociones de Bildung o Bildungsroman. Cuando se teoriza, por otro lado, sobre su condición como Bildungsroman, el aspecto temporal no supone más que un accesorio; no exhibe la importancia que creemos que debería tener. Ambos acercamientos, de este modo, con frecuencia, no reparan sobre la centralidad del componente temporal y durativo en la novela de Goethe: la presencia del tiempo en el título no debería

1 Aunque todavía no existan traducciones al español de los análisis de tiempo y narración de Müller, Hamburger o Harald Weinrich, el tercer volumen de Tiempo y narración de Paul Ricœur, en su misión de conectar filosofía y estudios narrativos, además de recuperar la argumentación de Benveniste y el aparato conceptual de Genette, ofrece un correcto resumen a sus conceptos. Cfr. "Los juegos con el tiempo" (Ricœur, 2008: 469-532) y también "Tiempo" (Martínez y Scheffel, 2011: 51-73). 
tenérsela por algo azaroso. El proceso de aprendizaje sería un proceso durativo, temporal, medido en y que requiere años. Estos “años", que aparecen en la segunda y también en la planeada tercera parte (respectivamente, Los años de viaje y los aparentemente planeados “Años de maestría”) (Vedda, 2014: 168), ${ }^{2}$ exponen el carácter temporal, a su vez, de la noción tardo-ilustrada de Bildung. Aunque exista una diferencia de grado entre estas "nuevas" palabras, entre Bildung y Aufklärung, en la medida en que la primera, según Mendelssohn, incluye a la segunda (1784), ${ }^{3}$ puede considerarse a la Ilustración como un sinónimo, hasta cierto punto, de Bildung, al describir procesos de (auto)desarrollo que involucran al tiempo en un sentido durativo. Así, Kant describe la Ilustración con una metáfora temporal, como un proceso temporal: es la salida de la minoría de edad (Kant, 2013). Años, etapas, tiempo: los años de aprendizaje serían entonces los que conducen a la madurez y la autonomía del adulto.

Sin embargo, el Bildungsroman no deja de ser una novela; tiene, por ende, como causa necesaria las mismas transformaciones epistemológicas y expone, aunque sea parcialmente, las mismas características generales de la forma novela. ${ }^{4}$ Por tanto, lo que aquí se sugiere es poner en contacto ambas dimensiones, los análisis narrativo-temporales con los rasgos específicos y textuales del Bildungsroman: en cierto sentido, es continuar la línea iniciada por Bajtín en su inconcluso trabajo, con el fin de aprehender concretamente las singularidades, la especificidad que presenta el Bildungsroman en su configuración temporal, que, además, dista de ser homogénea o repetitiva, en cada realización de este subgénero. Mostraremos la relación especifica del Bildungsroman con el tiempo atendiendo a tres aspectos de Los años de aprendizaje de Wilhelm Meister: la construcción de elipsis y su relación con el olvido; la idea de visualización del tiempo presente; y, por último, la figura del joven como individuo definido por el tiempo. Así, tiempo y formación se imbricarán al desplegar estos tres elementos. En definitiva, a diferencia de anteriores abordajes, analizaremos la estructura temporal de la novela de Goethe en tanto Bildungsroman.

Antes de relevar las acertadas observaciones de Bajtín, señalemos una particularidad en la recepción crítica de la novela de Goethe. Lecturas contemporáneas

2 Más allá del deseo de hacer explícita la referencia (que se satisfacía con el nombre del héroe), es interesante que la secuela apócrifa de Johann Friedrich Wilhelm Pustkuchen (1793-1834) de la novela goetheana conservase como principio constructivo el tiempo en su título, incluso agregando en una de sus partes otro tipo de organización temporal: la del diario (Wilhelm Meisters Tagebuch).

3 En "Ueber die Frage: was heißt aufklären?", texto que precede por dos meses a la conocida respuesta de Kant, Mendelssohn considera a la Bildung como el objetivo último, superior, del desarrollo humano y nacional. La Bildung estaría así compuesta por Aufklärung y Kultur. La formación comprende así un proceso de Ilustración, por medio de las ciencias, y uno de aculturación, a través de la sociabilidad, la poesía, y la elocuencia, respectivamente.

4 La experiencia del tiempo en las novelas de formación responde, en mayor o menor medida, al modo de representación temporal de la forma "novela", de las características y rasgos de esta forma literaria, cuya emergencia supuso una manifestación literaria del quiebre o transformación epistemológica que da inicio a la Edad Moderna. Pues, como sostiene Watt, el mundo de la novela es un mundo de individuos particulares teniendo experiencias particulares en tiempos y lugares particulares (1957: 31); individuos que también tienen una noción particular y temporal del yo, una identidad personal que se construye en el curso del tiempo o, mejor, en la duración. La dimensión temporal cobra importancia como proceso, como curso de acciones y eventos, como punto de ingreso de la vida cotidiana (como analiza Moretti, 1985) frente a la atemporalidad o circularidad del tiempo en formas literarias anteriores a la novela, como el tiempo concentrado del teatro (en oposición al expansivo de la epopeya). 
a su publicación, como la de Humboldt, o ligeramente posteriores, como la de Morgenstern, son testimonios de una recepción que veía en la novela goetheana una representación correcta de su época y contexto: en una epístola, el primero afirma que el gran mérito de la novela radica en su descripción "[d]el mundo y la vida tal como son, completamente independientes de un solo individuo y, por lo tanto, abiertos a cada individualidad"; mientras que el segundo -quien acuña el término Bildungsroman- considera, en 1820, que Los años de aprendizaje muestra las costumbres alemanas de la época (Steinecke, 1991: 74, 80-81). ${ }^{5}$ La crítica de Novalis a la novela puede comprenderse en este sentido también, al reprochar su excesivo apego a la vida prosaica, a la realidad ordinaria y burguesa (Novalis, 1978: 800).

Son lecturas que ven un fuerte contenido realista o mimético en la novela y que, por tanto, parecen encontrar una coincidencia entre el tiempo de la novela y su contexto de publicación. Sin embargo, la novela ha sido sujeta a lecturas que niegan o restringen la importancia del componente temporal, tanto del contenido como de su forma. En esta línea, posiblemente, el exponente más prestigioso -pero no el único- sea Auerbach, quien, a pesar de considerar Los años de aprendizaje como una de las obras más realistas de Goethe, registra un déficit en su representación de la realidad, en gran parte por su representación temporal. ${ }^{6}$ En efecto, habría en la novela cierta ambigüedad: a simple vista resulta difícil verificar en Los años de aprendizaje determinaciones específicas sobre el espacio y el tiempo "real", histórico. Comparada con novelas inmediatamente anteriores y posteriores a ella, su representación del tiempo es singular.

$\mathrm{Al}$ analizar la imagen del héroe en los Bildungsromane, Miles (1974) describe a Wilhelm Meister como un personaje "picaresco", rasgo que caracterizaría, a su vez, a la novela en general. Uno de los argumentos de Miles, sino el principal, para argumentar esta configuración picaresca -en oposición a la que reconoce en novelas posteriores, más cercanas a discursos de "confesión", como Enrique el verde, de Keller, o Los cuadernos de Malte Laurids Brigge, de Rilke- es, nuevamente, la presunta atemporalidad de la novela. Wilhelm Meister, en tanto héroe pícaro, no cambiaría, no modificaría su imagen de héroe; en este sentido, por tanto, no existiría Bildung alguna en el héroe del Bildungsroman por excelencia. Incluyéndose en la tradición de autores como Jacobs (1981), que cuestionan la realización formal y textual de la noción de Bildung, hasta el punto de discutir la existencia del Bildungsroman en tanto tal, Miles plantea que la Bildung se realizaría parcialmente en la figura del narrador goetheano (que aún estaría limitado por la tercera persona), pero no en Wilhelm. Es decir, usando los conceptos de Müller (2014), se daría un proceso de formación en el tiempo de la narración (Erzählzeit) pero no en el tiempo narrado (erzählte Zeit), porque el mundo de Wilhelm y su

5 Traduzco del alemán. La fuente es una carta del 24 de noviembre de 1796 de Humboldt a Goethe.

6 Para Auerbach, el "campo de lo real" en la novela está limitado porque el tiempo y el espacio son descritos de un modo demasiado general y la sociedad parece inconmovible, inmutable: "El mundo clasista burgués reposa ante los ojos del lector en una calma casi intemporal" (1996: 420-421). Sammons expresa, acertadamente, su desacuerdo al señalar que la novela no carece de un pronunciado sentido del tiempo: a través de una serie de indicios (como los debates sobre la necesidad de un teatro nacional), la novela se sitúa de manera reconocible en el último cuarto del siglo XVIII (1991: 38). 
modo de relacionarse con este -en coincidencia con Auerbach- estaría marcado por el detenimiento, por la atemporalidad.

Sin embargo, es excesivo negar todo cambio o transformación de Wilhelm Meister; más bien, el desarrollo del protagonista no se encuentra tan pronunciado, es más sutil, en la medida en que responde a la estética goetheana que renuncia a las posiciones extremas. Y es esta construcción sutil, que no establece su relación con el tiempo histórico con un sistema de datación explícito, con fechas precisas, la que complejiza la experiencia y representación del tiempo: en la novela parece predominar un tiempo pleno, en algún punto ideal, que no es ni un pasado biográfico y continuo ni (completamente) el desarrollo de un futuro utópico. Si la Ilustración supone un mayor distanciamiento entre los tiempos, un quiebre con la recursividad del futuro (Steiinby, 2017: 15); entre el tiempo pasado que prefigura el presente, y el futuro a construir, en tanto horizonte posible, Goethe, entonces, parece construir "la plenitud del tiempo" (Bajtin, 2013: 232) sobre estos puntos intermedios, en el espacio entre los tiempos.

No es, entonces, la atemporalidad de una representación realista, incompleta o fracasada, ni la de la picaresca; pero, que se pueda argumentar esto más o menos convincentemente demuestra lo elusivo y singular del tiempo narrado y el tiempo de la narración para el tiempo del que observa o lee, sujeto al tiempo real, de la vida (Zeiterlebnis) (Müller, 1974): en cierto sentido, la plenitud del tiempo, el presente pleno que se da en el juego entre narración y relato, habilita para el personaje, pero también para el lector, una conjura de olvido, a través de la ironía.

\section{El tiempo del olvido}

La novela comienza con un héroe en contacto con el pasado, con las experiencias pasadas, ya desde su primera aparición. El incipit de Wilhelm, su primera línea de diálogo, es una afirmación de haber oído (antes, en un momento previo al tiempo narrado) los cuestionamientos de su padre sobre su interés por el teatro. A esto le sigue el recuerdo de la infancia. El vínculo con el pasado es fuerte y se manifiesta en este recuerdo de infancia, donde se gesta la vocación disruptiva. El pasado también es memoria y es el Wilhelm del primer libro el que se hunde en el recuerdo de su amada actriz, Mariane, del que se debe sustraer, ayudas mediante, con actividad. El principal ayudante es justamente Werner, su amigo, el personaje del comercio, más ligado al mundo activo, al mundo del trabajo burgués.

Precisamente, es en el pasado del tiempo narrado donde opera con mayor insistencia la figura del narrador, esto es, donde hallamos un mayor desfasaje entre el tiempo narrado, diegético, y el del tiempo de la narración, el construido y presentado por el narrador. Müller utiliza el concepto de "contracción del tiempo" (Zeitraffung) (Müller, 1974), pero bien podríamos apelar, como también hace este 
autor, a la noción de Thomas Mann de Aussparung, ${ }^{7}$ de "vacíos", "huecos" en la narración. Detrás del acto de producir estos “vacíos”, de condensar el tiempo narrado -más allá de la obvia imposibilidad de reproducir la cadencia del tiempo de la vida-, existe una decisión estético-narrativa que aquí, por la noción de Bildung, asume también un carácter ético. El narrador goetheano decide contraer el pasado que, como veremos, conspira contra la Bildung $y$ la felicidad (Glück) del héroe.

La relación de Wilhelm con Mariane termina abruptamente cuando el joven descubre a la actriz con otro hombre. El narrador, sin embargo, omite deliberadamente la secuela de esta separación. En efecto, los años posteriores a este súbito final, cuya cantidad no define, son resumidos -en pocas oraciones de una patente generalidad-como un período marcado por la melancolía. Es un período de tiempo, según la reflexión sobre esta elipsis del consciente narrador goetheano, que no interesa en la medida que comprende años de un proceso clausurado, agotado:

Todo el que ante nuestros ojos lucha por un objetivo siempre despierta nuestro interés, independientemente de que alabemos o deploremos su meta. Mas cuando ya su lucha ha alcanzado un desenlace definitivo, apartamos de él la vista. Todo lo que ha acabado y rematado difícilmente puede volver ya a cautivar nuestra atención, especialmente si le augurábamos un fin desastroso a su empresa (Goethe, 2019: 155).

El narrador, pues, recorta, selecciona años específicos para la narración: son años, no la vida de Wilhelm Meister; selección que, como vemos, está cargada de significado y que encapsula no solo una decisión estética (lo narrado es narrado porque es interesante, es poético, es bello, etc.) sino también, como fue mencionado, una ética (se contrae la narración en virtud de su utilidad, provecho o valor para el héroe y para el lector). Este acto de selección, por lo demás, no se limita a Los años de aprendizaje; está, en efecto, aún más acentuado en Los años de viaje de Wilhelm Meister, su continuación, donde la estructura de epístolas, Novellen y aforismos, de archivo, con una narración menos focalizada, esboza elipsis más extensas, contracciones temporales más fuertes: es imposible de relevar completamente, ya sea en su recorrido interior o exterior, la vida de Félix. Ambas novelas comparten esto, pues, porque en ningún momento pretenden esbozar una biografía completa o exhaustiva del héroe; algo que, por otro lado, diferencia tanto a ambas novelas de los Bildungsromane posteriores como Enrique el verde, más próximos a la escritura autobiográfica, que operan con categorías temporales más amplias y de una continuidad más clara que el tiempo narrado y el tiempo de la narración goetheano. ${ }^{8}$

7 "Por cierto, nada tenemos en contra de la escisión [die Aussparung]. Es bienhechora y necesaria, pues a la larga es imposible contar la vida tal como ella se contó a sí misma en otro tiempo" (Mann, 1960: 702).

8 Este mayor grado de continuidad temporal tiene un correlato en la estructura lógica de la novela, en las relaciones causales de sus eventos: una narración menos elíptica con frecuencia produce cadenas de causaconsecuencia más comprensibles que las que advertimos en la vida de Wilhelm, la cual, en parte también por su carácter, parecería exhibir cierta falta de motivación causal. En este sentido, la novela parece responder más a una "motivación compositiva o estética" al seguir criterios estilísticos (o, aquí, estético-éticos) y no empíricos o causales (Martínez y Scheffel, 2011: 165) 
Tenemos, entonces, un narrador que, a través de esta específica selectividad, de estas operaciones de narración y contracción del tiempo narrado en la estructura narrativa, parece apostar por relacionar el carácter olvidadizo y, en parte, receptivo-maleable de Wilhelm Meister con la predisposición que debería asumir el lector. ¿ ¿Quizá es así como entra en contacto la Bildung del relato con la experiencia del lector? ¿Es así como debemos entender la complicidad que instaura el narrador con su permanente ironía? La reiterada descripción del humor de Mariane y Bárbara, su vieja ama de llaves (aburridas, distraídas, somnolientas), frente al "interminable relato" de niñez de Wilhelm Meister en el primer libro (Goethe, 2019: 103), en el que relata (en primera persona) el inicio de su pasión por el teatro, puede entenderse, de este modo, como una interrelación entre narración, ironía y una actitud particular frente a la experiencia pasada. Análogamente, está el comentario del narrador en el cierre de estos recuerdos, cuando retoma la narración, donde advertimos aquella ironía como principio estructural del Bildungsroman, esa “necesaria ambigüedad” señalada por Lukács (2002: 141) o autoconciencia epistemológica, para Swales (1991: 66):

Por la intensidad de su abrazo y por la viveza de su voz, Mariane se despertó y escondió con dulces caricias su turbación. No había escuchado ni una sola palabra de la última parte de aquel relato y es de desear que las anécdotas favoritas de nuestro héroe encuentren en el futuro lectores más atentos (Goethe, 2019: 112).

A lo largo de la novela, libro tras libro, Wilhelm atravesará la distancia que media entre pasado y presente bajo el filtro constante de la ironía del narrador; es un camino de desengaño y de olvido. Wilhelm olvidará a Mariane definitivamente con la muerte de Mignon -quien, con su muerte, además, se lleva los mementos de Mariane-, y asumirá la posición de un hombre de presente pleno, o, más bien, presente vivo, que no es un presente corto de miras, alienado. Steiinby dice que los individuos en la novela se explican a través de las acciones que realizan en el tiempo (2017). Bajtín, por su parte, reconoce la capacidad de Goethe para leer el tiempo en las actividades, en el espacio, haciendo visible el tiempo histórico. Goethe quiere ver los "vínculos necesarios del pasado con un presente vivo, quiere comprender el lugar necesario que ocupa el pasado en el proceso permanente del desarrollo histórico" (Bajtín, 2013: 222); es decir, la "necesariedad" del pasado, la plenitud del tiempo; y no el pasado enajenado, pasado en sí y para sí, que sería el tema predilecto de los románticos. Esto es, queda en el olvido aquello que ya no aportaría nada al proceso formativo (como los años en el teatro) o que supone un obstáculo para la felicidad del héroe (como el recuerdo de Mariane).

Wilhelm se estaría despojando de este pasado de ruinas, de este pasado que está muerto, que no es productivo al presente ni al tiempo que la novela vislumbra hacia el final: el futuro. En esta clave puede leerse el sueño del protagonista en el

9 Humboldt, en su carta a Goethe, también reconoce esta especificidad del carácter de Wilhelm, diciendo que este posee una "capacidad de determinación universal [Bestimmbarkeit], mas casi sin disposición auténtica" (Steinecke, 1991: 74). Cfr. nota 4. 
séptimo libro. El sueño evoca el pasado: en un jardín que de niño había frecuentado, Wilhelm se reencuentra con Mariane, a la que aún cree viva. Pero la breve conversación que mantienen -que el narrador no refiere- se ve interrumpida por la aparición del -para este punto de la historia- difunto padre de Wilhelm, quien aparta a Mariane del joven. Wilhelm trata de seguir a ambos personajes, mas lo eluden, hasta que, eventualmente, alzan "vuelo" y desaparecen de su vista. A Wilhelm, "[l]a naturaleza y el amor le indujeron a volar tras ellos [su padre y Mariane], pero la mano de la amazona le sujetaba" (Goethe, 2019: 506). Lo retiene un personaje con vida: es Natalie, la mujer ideal y real que le salvó la vida en sus años como director y actor de teatro, cuyos lazos con el tiempo presente la distinguen del padre de Wilhelm y, sobre todo, de la figura de Mariane. El pasado, por tanto, tiene que mantener una relación necesaria, viva, con el presente, sino, el olvido. Así, el olvidadizo Wilhelm pide que no le recuerden su época teatral a la par que el narrador condensa el pasado del héroe en un pergamino, separado de su cuerpo individual, y suprime a los personajes más poéticos, más románticos (Agustín, Mignon, Aurelia, también el “Alma Bella”), que por su conexión con el pasado y por su falta de renuncia (Entsagung), no poseerían vida ni en el presente ni futuro.

La proyección a futuro en la novela queda reservada a los personajes que no se encuentran limitados por un tiempo en específico, sea el pasado o el presente materialista, que es capitulación precipitada a la realidad del rédito, como vemos en Serlo, Milena y, principalmente, Werner. Los personajes que están, por así decirlo, abiertos temporalmente, que existen en el presente vivo, tienen futuro, tienen la posibilidad de superar (¿y completar?) la Bildung individual en pos de una formación en comunidad, colectiva, más o menos exitosa, más o menos satisfactoria; pero, principalmente, parecen tener derecho a alcanzar el objetivo -que inserta a la novela de Goethe en el contexto más amplio de las novelas del siglo XVIII- de una felicidad real, plena.

"[L]a máxima de la novela pareciera ser [...] que el secreto de una vida feliz y efectiva reside en no tener una muy buena memoria" (Koval, 2018: 168); en tener, pues, una relación particular con el pasado, que involucra contracciones temporales en la narración y el olvido en el sujeto: "No me recuerde aquellos tiempos en un momento de tan gran dicha” (Goethe, 2019: 691). Así, en comparación con novelas previas, como Anton Reiser, de Moritz (novela que el Wilhelm Meister "corrige"), y posteriores, como Enrique el verde, donde el pasado ingresa permanentemente a través del pretérito de la escritura autobiográfica, aquí "la hipertrofia del recuerdo se cambia por el 'don' del olvido” (Koval, 2018: 313).

Especial atención, entonces, debe de prestar el héroe a su relación con el tiempo; lección que el narrador parecería querer replicar en el lector con la disposición del tiempo de la narración; y la consciente decisión de la tercera persona por sobre la primera, la cual tendería más a la meditación y reflexión sobre el pasado, al ensimismamiento, como vemos en dos momentos: en la descripción que Wilhelm hace de su infancia y en las "Confesiones del Alma Bella". 


\section{La visión del presente}

Si por el lado del pasado, tenemos el olvido, un olvido que como vimos, supone un proceso de selección y exclusión, de "escisión" para Mann; por otro, tendríamos aquella singular presentación del tiempo narrado y tiempo de la narración, que llevó a Bajtín a hablar de tiempo pleno. Para Bajtín, Goethe es al mismo tiempo heredero y conclusión de la Ilustración: Goethe alcanza la cumbre de la visión artística del tiempo histórico, que, en ciertos aspectos no ha sido superada, por su configuración temporal en la novelística (2013: 213). El tiempo pleno sería, así, la capacidad cronotópica de Goethe, su capacidad de ver el tiempo en el espacio, de percibir "la relación visible y esencial entre los tiempos (el pasado en el presente), el carácter creativamente activo del tiempo (del pasado en el presente y del presente mismo)" y "la inclusión del futuro que concluye la plenitud del tiempo" (Bajtín, 2013: 231). Podríamos decir, en otras palabras, que no es ni un tiempo biográfico ni uno pícaro, cíclico, sino que es el del optimismo que no podrá volver a ser igualado (Moretti, 1985: 116); el preciso tiempo de la Ilustración y de su posible superación, que va del individuo ilustrado hacia la comunidad, el género humano.

A esta evaluación se puede añadir el comentario de Müller sobre el acto de narrar como efecto de visualización (vergegenwärtigende Wirkung), representación de algo que ha sucedido y modo de hacer presente algo que está ausente (Müller, 2014: 250). En conjunto, en consonancia con la importancia que tiene lo visual para el pensamiento ilustrado y, específicamente goetheano, aquí la narración visualiza el tiempo, aquellas relaciones esenciales entre los tiempos. Precisamente, es lo que sucede en Los años de aprendizaje: el narrador hace perceptible el tiempo, que es tiempo presente y vivo. ${ }^{10}$

Recordemos la medida que puede curar al arpista de sus males. El melancólico arpista Agustín, quien desde temprano en la novela viaja junto a Wilhelm, aquejado por un pasado que lo vincula a otro personaje de un modo siniestro, tras una situación que pone en duda su cordura, es internado en el campo, bajo el cuidado de un pastor y un médico. El método de sanación que aplican involucra el tiempo. El pastor le explica a Wilhelm que "[ha] compartimentado las horas del día del anciano". Al arpista se le organiza la jornada del día con actividades varias, se dispone de su tiempo de modo que la ociosidad no habilite el ensimismamiento en el perturbador pasado que, en palabras del médico que lo supervisa, sería "una idea fija que no contribuy[e] a la vida activa" (Goethe, 2019: 424). O, sino, consideremos el acto de escritura objetiva o distanciada, de la propia vida, bajo la forma de un pergamino, con los “años de aprendizaje" del protagonista: es otro modo de visibilizar el tiempo, de darle, en este caso, en particular, una forma concreta, espacial, fuera del sujeto; $y$, por tanto, no constituye carga ni culpa alguna el pasado para el héroe.

10 Esta idea de visualizar el tiempo puede pensarse como una característica de las novelas de formación en general, siempre que se asuma una posición teórica más amplia sobre el Bildungsroman, que encuentre este subgénero en otras literaturas, como hace Moretti $(1985 ; 1987)$. Es, después de todo, fascinante la observación de Müller sobre la datación temporal en los capítulos de Tom Jones de Fielding, a quien él considera como el fundador de la novela de desarrollo (Entwicklungsroman) (2014: 252). 
En ambos casos, el acto de volver visible el tiempo, el tiempo presente que puede ser promesa de un final feliz, requiere de un agente externo que organice y presente la materia en la forma adecuada. Cuando Lukács habla de "atmósfera de seguridad" hace referencia a cierto aspecto artificial en la novela (de Märchen incluso) que permite que el héroe se inserte exitosamente en la sociedad o, por lo menos en la comunidad de la Sociedad de la Torre (Lukács, 2002). Y si dentro de la novela esto se ve en la Sociedad de la Torre, el correlato en la escena del tiempo de la narración es el narrador, quien desempeña un rol específico: el rol del director. Adorno, en un texto sobre Goethe y el clasicismo alemán, describe el accionar goetheano (que aquí aplicamos a su narrador) como "un logro del tacto": "Mediante la apariencia de naturalidad esconde la mano del director de escena, que da sentido" (Adorno, 2003: 483). Este acto de equilibrar allí donde no hay reconciliación, según Adorno, es una solución frágil, que alcanza un precario punto medio a través del recurso del lenguaje, de la sutil construcción de sus obras. Entonces, sin perdernos demasiado en los juicios adornianos, el narrador como director construye una "escena" visual, un significado, con un determinado objetivo de cara a su recepción.

El tiempo de la narración no aspira a sustituir el tiempo de la vida, no intenta reproducir el tiempo histórico o real: no busca la naturalidad, sino la construcción de sentido. Müller, en su argumentación, utiliza un ensayo de Goethe que es dable recuperar aquí, no solo por su proximidad temporal, sino también por su relevancia: es correcta la observación de Salmerón sobre la presencia de la teoría de la mera apariencia en el cuarto capítulo del primer libro de Los años de aprendizaje (Goethe, 2019: 96). ${ }^{11}$ Publicado en 1798, el comentario de Goethe al ensayo de Diderot sobre la pintura (Diderots Versuch über die Mahlerei), pues, contiene unas reflexiones sobre el hecho artístico que -continuando con lo planteado por Müller- pueden ser puestas en relación con el arte de la narración. Allí encontramos la sugestiva formulación sobre el artista como productor de una segunda naturaleza, ${ }^{12}$ que no está viva, pero que ha sido perfeccionada por el hombre; es un objeto reflexivo, cargado de significado por el artista y que, luego, a partir de lo que está presente en la obra de arte, es reconocido por el observador (Goethe, 1991). ${ }^{13}$

Müller traslada esta observación estética al campo de la narración, considerando que, en el caso de la producción poética, es el narrador quien articula el objeto natural, vivo e indiferente, y le otorga significado humano a la naturaleza; en definitiva, es quien presenta al lector la mera apariencia, el objeto ya construido, la naturaleza distinta (Müller, 2014: 263). El narrador consigue esto por medio de una delicada construcción narrativa, de determinadas operaciones, articulaciones sobre el tiempo: a través de la narración explícita de ciertas secuencias

11 Cfr. nota 3 de Salmerón de op. cit.

12 Es más preciso hablar de una naturaleza distinta, o una naturaleza otra (andere Natur). No debe, pues, confundirse con la "segunda naturaleza" lukácsiana -y, posteriormente, con revisiones, adorniana- de Teoría de la novela, concepto que refiere a otro producto artificial, humano: el denominado mundo de la convención, la sociedad e historia humana, inaferrable e incognoscible.

13 Otra formulación aparece en una máxima de Goethe: "Arte: una naturaleza diferente, también misteriosa pero más comprensible porque surge de la razón" (1982: 467). 
temporales, consideradas importantes para la producción de sentido y la omisión (o contracción) de otras, cuya presencia interferiría en la recepción estética (Müller, 2014:266). Entonces, lo que llamamos aquí el acto de visibilizar el tiempo es, a su vez, un modo de cargar de significado, ya sea por narración o elipsis. Es así como el narrador-director goetheano puede hacer coincidir el efecto del tiempo de la narración, el tiempo narrado y, atendiendo a su carácter de Bildungsroman, el tiempo de la formación.

Y es que Los años de aprendizaje de Wilhelm Meister se encuentra socio-históricamente en el tiempo justo de la Bildung; en el tiempo idóneo del concepto de formación, "el momento justo" [Es ist eben zur rechten Zeit] (Goethe, 2019: 586). Como Lukács sostenía, el mundo de esta novela es un mundo aún no desencantado en su entereza, que aún puede tener significado para el individuo y donde la posibilidad de un futuro esperanzador, de mejora social -aunque no sea por vías estrictamente revolucionarias- no se ha perdido ni corrompido por la evolución del capitalismo. Si, continuando con Lukács, en la novela de Goethe, hay un equilibrio entre el alma y el mundo, irrepetible en el desarrollo de la sociedad burguesa, de un modo análogo, se puede pensar que Los años de aprendizaje se encuentra en -y construye en la narración- el punto de equilibrio, el punto justo donde la experiencia del tiempo no es problemática ni se halla limitada.

Por su visualización del tiempo pleno, por construir y representar un tiempo (ideal) de la formación, la novela de Goethe parece mostrar una vida social aún abierta a diversas vías de desarrollo, ${ }^{14}$ con frecuencia, sí, utópicas, aunque siempre matizadas por la narración irónica, que otorga un fuerte elemento autoconsciente a la recuperación de los debates de su propia época sobre la noción de Bildung, sobre la posibilidad de una alternativa futura a través de la educación estética, de la cultura. Se trata de discusiones que, en sí mismas, eran fenómenos en gestación en la época, más allá de que hayan permanecido como promesas sin realización o cuya materialización supuso acentuar lo represivo de su origen ilustrado, la dialéctica de la Ilustración. Y esto lo podemos ver también a través de otra figura ligada a la dimensión temporal: es la figura del joven y el desenlace que debe (o no) tener el período de juventud.

\section{El proceso de la juventud}

En la Modernidad, el joven, la juventud -que en gran parte comprende el nuevo sujeto histórico del adolescente- cambia sus coordenadas, pasa de la determinación espacial a una determinación principalmente temporal (cfr. Gillis, 1981; Roseman 2003). Así, la juventud pasa a ser un problema, en la

14 Auerbach concluye que Goethe nunca ha representado la realidad de la vida social coetánea dinámicamente, "como germen de fenómenos futuros o en gestación" (1996: 423), aunque, desde una perspectiva que no pretende toparse con las características del realismo decimonónico europeo (específicamente francés) en una obra que pertenece más a las discusiones histórico-estéticas del siglo XVIII; desde un análisis que se ocupa en reconocer la especificidad de la novela goetheana y del subgénero que -accidentalmente o no-funda y que la adopta como modelo, difícilmente pueda afirmarse lo mismo. 
medida en que ya no se puede sostener el viejo concepto de lo joven, asociado a la repetición, al camino predecible, a la idea de sucesión y herencia de una tradición o profesión. Ahora, lo joven acentúa el dinamismo, la inestabilidad de la Modernidad; es, según Moretti, su esencia, "el signo de un mundo que busca su significado en el futuro, en lugar del pasado” (1987: 5). Identificado con la Modernidad (y el Bildungsroman como forma simbólica de la juventud y la Modernidad), el sujeto joven asume sus características: la indeterminación, lo informe, la movilidad.

Pero, el hecho de volverlo una forma, de narrar el proceso de exploración y desarrollo del joven supone la imposición de un límite a esta indeterminación: youth does not last forever. Es decir, la forma literaria (la novela) le da una idea de cierre a lo joven, conjura estéticamente -crea confort según Moretti-el horror de la Modernidad: la impredecibilidad, el dinamismo, la velocidad que trae aparejada la obsolescencia. Esto le daría al Bildungsroman una estructura contradictoria, paradójica, que operaría entre opuestos, entre el dinamismo y los límites, entre la apertura y el final; entre, en definitiva, lo joven informe y el joven formado. Así, aunque la aseveración sobre el principio clasificatorio en la novela goetheana parezca excesiva (en tanto que la novela no parece postular un tipo de vocación y camino formativo tan unilateral y normativamente como piensa Moretti), si es cierto que, en Goethe, la juventud debe subordinarse, en mayor o menor medida, -agreguemos, en el momento justo- a una noción de madurez, que estaría más cerca de la felicidad y de una noción de identidad más estable. Es decir, se concibe la juventud, entonces, como una fase, un período de transición; un proceso que tiene un objeto, un final, y que, por tanto, no tendría valor en sí mismo, sino solo por su carácter relacional. La encrucijada de los adolescentes, -haciendo referencia a la única obra dramática terminada por Wilhelm- debe tener una salida.

En la estructura narrativa, esto lo vemos, desde ya, en la decisión de contraer ciertos episodios, pero también en la representación de los personajes, en tanto pueden ser caracterizados a grandes rasgos por una afinidad con un tiempo en particular. Al referirnos al olvido, adelantamos que en la novela habría personajes más sujetos a un tiempo, fijado (parafraseando al médico de la novela) a una terrible e improductiva idea; mientras que existen otros más abiertos, tanto a la prospectiva felicidad como respecto al tiempo. En efecto, la novela tiene un carácter polifónico y la ironía contribuye a su "necesaria ambigüedad", donde "se encuentran lo bastante repartidas las medidas de verdad y equivocación como para que no sea posible identificar una perspectiva como la correcta" (Vedda, 2014: 142) y no hay imposición de una respuesta unívoca. Pero esto no supone que no sea posible distinguir posiciones que son representadas más negativamente que otras, creándose un ensayo de orden en lo múltiple, por lo menos, narrativamente, en línea con la idea de artificiosidad marcada por Adorno. Esto es, si bien se reproduce la multiplicidad y la falta de solidez de la Modernidad, se elude el relativismo absoluto: no se hallan difuminados de manera completa los rasgos positivos y negativos, aún es posible establecer, con mayor o menor dificultad, las acciones que, narrativamente, son consideradas como positivas (exitosas) o 
negativas (fallidas); o el orden que parece imperar entre los personajes en relación al éxito de su formación (es más positivo un personaje como Natalie que Mariane o Therese en comparación con Madame Melina, por ejemplo).

Esto no supone que los sujetos más relacionados a aquel tiempo pleno, el presente vivo (y visible), sean individuos que permanecen en el estado de juventud, en el estado concebido como transitorio. Al contrario, son estos personajes los que instauran (asistidos por el narrador) un objetivo y un fin a este período; los que pueden, dichosamente, trazar una línea, casi completamente arbitraria, de final del tiempo de juventud, de aprendizaje: por esto mismo, la afinidad temporal y la posibilidad del happy-ending se confunden, al ser, en efecto, los mismos personajes los que acaban casados hacia el final de la novela. En estos personajes, incluso en los menos serios, como Friedrich y Philine, persiste una idea de clausura de la juventud: bien por contraer matrimonio, por asumir un rol de padre o madre, $o$ por la toma de acción política-pedagógica (futura). ${ }^{15}$ Más allá de que no sean precisamente realizadas en la novela y queden como un evento a desarrollar (como la vocación de Wilhelm), estas acciones terminan definiendo a los personajes, los asocian al tiempo vivo y los alejan de las capitulaciones filisteas del presente burgués o el rechazo romántico más extremo, que conduce hacia el pasado.

Planteado de este modo, puede argumentarse que los personajes que se asocian a la problemática interiorización y fijación en el recuerdo, como Aurelia o Agustín, son los sujetos que permanecen voluntariamente prendidos a una idea de perpetua juventud. Tomando una de las premisas metodológicas de Koval, estos personajes permanecen en el "estado marginal o liminal" (Koval, 2018: 21-22), que, para nosotros, comprendería también una alienación o incomodidad en el tiempo. Estos personajes no solo no reingresan (o se agregan) a un estado de normalidad, no renuncian ni se reconcilian con la sociedad, sino que tampoco pueden vivir en el tiempo presente. No pueden existir plenamente en su época, como vemos en el destino fatal que les construye el narrador: la muerte temprana, el no poder acceder al happy-ending, supone una especie de condena de estos personajes más poéticos, verdaderos modelos de fracasos formativos. Es por este rol de ejemplo negativo por el cual no hay contracción temporal respecto a sus muertes; incluso, la vida de uno de estos personajes tiene un libro entero: las "Confesiones de un Alma Bella".

El Alma Bella es un personaje curioso, que exhibe concretamente aquella polifonía de matices de los personajes goetheanos: posee cualidades positivas, pero, de nuevo, por obra de la ironía en la construcción, tanto del tiempo narrado como del tiempo de la narración, acumula una serie de rasgos negativos. En primer lugar, en el tiempo narrado, su biográfico y sumamente reflexivo escrito, que remite desde título al género de las confesiones, principalmente, a las Confesiones de

15 "Si hubo un elemento de 'retomo a' en mi itinerario intelectual, fue [...] una ampliación del campo de formación [Bildung]. Quería envejecer. Quería convertirme en alguien de mucha edad, reclamaba encarnar tres mil años de historia europea, según la recomendación de Goethe" (Finkielkraut y Sloterdijk, 2008: 13; la cursiva es nuestra). Sloterdijk destaca, casi casualmente -gesto que, por extensión, repetimos aquí-, un aspecto significativo: el ideal de madurez y de vejez implícito en la noción de Bildung. 
Rousseau, es un fracaso: es un texto inútil. No salva a Aurelia de su melancolía, ni logra mellar en Wilhelm, más allá de informarle acerca de la filiación que une al Alma Bella a otros personajes más positivos, como Lothario o Natalie, quien es, en esencia, ella si hubiese vivido realmente y no solo para sí; algo que, por lo demás, la misma autora reconoce al describir la admiración que siente por Natalie. Su excesiva subjetividad no logra crear, pues, un efecto positivo en sus receptores, al no disponer de un modo de comunicación efectivo con el exterior: no hay que olvidar que el título es elegido por el médico, lo que indica su condición de texto privado, que no fue escrito con un objetivo de publicación, ni, quizá, de lectura alguna más que la propia. Por otro lado, en su contenido en sí están los aspectos negativos que sintetiza Natalie: "Una salud quebradiza, una tendencia excesiva al ensimismamiento, una inquietud moral y religiosa demasiado constante", todo lo que la volvió un sujeto limitado, que brillaba para pocos (Goethe, 2019: 598-599).

En segundo lugar, está el tiempo de la narración, que incluye su ubicación en la novela. El posterior contraste, tan próximo a este libro, con el pergamino de los años de aprendizaje de Wilhelm, que fue escrito por la Sociedad de la Torre, sin sucesos aislados ni sentimientos anecdóticos, no parece hacer más que resaltar el peligro del ensimismamiento y la auto-escritura para la formación del individuo. Es más, se nos dice que el escrito sobre Wilhelm no lo proyecta como un espejo, sino que ofrece un "retrato" a partir de su imagen (Goethe, 2019: 586); una segunda naturaleza, pues, realizada por el artista en contraste con el espejo del alma de una confesión que, además, supone un modo de comunicación más monológico, distinto al frecuente diálogo de la novela.

Por supuesto, en este nivel opera la elección del tiempo verbal, de la primera persona, y también está la mayor continuidad formal y temporal en su presentación, sin subdivisiones en capítulos y que va desde la temprana niñez hasta una suerte de reflexión cerca de la muerte. Tiene una función de receso, de bisagra entre el fin del mundo del teatro y la creciente centralidad de la Sociedad de la Torre: en cierto sentido, es un momento de distensión, autónomo y aislado de los demás libros, como su autora. Por último, el diálogo con el que reencontramos a Wilhelm tiene algo de aquella maliciosa ironía goetheana. Sobre cuánto tiempo estuvo con la compañía de teatro, Wilhelm le responde al cura católico:

\footnotetext{
-Más del que debí estar. Cuando me acuerdo de aquel tiempoy reflexiono, hallo un vacío inmenso; es un período que nada ha dejado en mí.
}

-Ahí se engaña usted. Todo lo que nos sucede deja huellas, todo contribuye, aun sin notarlo nosotros, a nuestra perfección, pero es peligroso intentar darnos cuenta, porque si nos la damos, o nos hacemos orgullosos o negligentes, o se apodera de nosotros el descorazonamiento o la pusilanimidad, y ambas alternativas son perjudiciales por igual (Goethe, 2019: 502).

¿Acaso es está la actitud que debe adoptar el lector frente a lo leído, frente a las "Confesiones de un Alma Bella”? ¿Tomarlas como significativas, pero olvidarlas, no meditar sobre ellas, de manera similar a lo que hace, pocas líneas después, Wilhelm respecto a la muerte de Aurelia? 
Estos personajes que no logran salirse de sí mismos, que no logran desenvolverse satisfactoriamente en la época en la que viven, en el presente vivo, que permanecen en una condición de marginalidad, de juventud que no logra alcanzar una efectiva y útil madurez (con matices, por supuesto, para un sujeto como el Alma Bella), tienen un contraste. Werner es el otro extremo, es la claudicación precipitada al presente, lo que da como resultado un sujeto que fracasa en su formación y en su desarrollo en el tiempo, por otra razón: se suprime la condición de marginalidad, la situación de juventud como sujeto de indeterminación. En otras palabras, Werner elude la etapa formativa completamente, lo que tiene como costo su integración a un presente chato, sin otro objetivo que el del rédito, y su envejecimiento prematuro, como el irónico narrador no duda en señalar. Es un adulto incompleto, que no tuvo juventud y cuyo futuro implica una repetición constante y árida del presente, del mismo estado de cosas. Si los personajes románticos permanecían en una postura informe, personajes como Werner o Melina se ubican en el polo opuesto, en lo estrecho, en la especialización excesiva que Schiller en su Sobre la educación estética del hombre, en una serie de cartas (1795) asemejaba a una mutilación; en, pues, lo deforme.

Entonces, dos extremos: uno ligado al pasado, al ensimismamiento, y el otro, a una apresurada capitulación a las exigencias del presente más grosero, more económico. Ambas posturas suponen ejemplos de fracasos formativos, de la imposibilidad de conciliar las contradicciones entre sujeto y sociedad, lo que redunda en una decisión unilateral a favor del individuo y lo interior por sobre la sociedad y lo exterior, o al revés. Esto tiene como correlato, pues, el problemático modo de relacionarse con el tiempo y de vivir en el tiempo de estos personajes, y, por tanto, la insistente representación negativa de estos sujetos frente a los individuos formados o con la posibilidad de formarse satisfactoriamente, que pueden cerrar un período de tiempo particular, a un período vital.

El mensaje para el sujeto joven sería el mismo que recibe Wilhelm, "[a]cuérdate de que tienes que vivir" (Goethe, 2019: 621), aunque con una observación. La juventud debe vivirse, pero debe terminar también: debe hacerlo en el momento adecuado, en el tiempo de la formación; de lo contrario, el joven corre el riesgo de no tener forma o de deformarse.

\section{Las novelas sobre el tiempo}

El olvido, la visualización de un tiempo determinado, la juventud. Estos fueron los tres elementos que desplegamos aquí, atendiendo a su realización narrativa en Los años de aprendizaje de Wilhelm Meister, a la par que establecimos líneas de interacción entre la dimensión temporal y la formación. Así, las contracciones temporales, las elipsis, constituyen una decisión estética y ética, que busca suprimir lo inútil y contraproducente a la formación del héroe, por medio del olvido, y del lector, a través de los vacíos de información. Por otro lado, el acto de volver visible, o más preeminente, un tiempo particular involucra la acción de 
un narrador-director que construye una realidad temporal ideal, pero no ingenua, para la Bildung. El narrador configura el tiempo de la formación, en el que se enfatizan las posibilidades de formación (y posterior acción) del presente en oposición a la negatividad del pasado enajenado, concluido y fuera del accionar humano, y la lejanía del futuro. Por último, la figura del joven emerge como un individuo temporal, definido y acotado por el tiempo, en tanto que la juventud es un proceso y período con un fin u objetivo, la madurez del individuo formado.

Sin embargo, como mencionamos, la importancia particular que cobra el tiempo rebasa a la novela de Goethe para abarcar, precisamente por su rol modélico, al Bildungsroman en general.

Aunque no explorado aquí, un factor clave en esta singularidad del Bildungsroman respecto a su dimensión temporal-narrativa se debe al contexto de emergencia de la noción de Bildung, contexto marcado por diversos factores: la recuperación y rápido crecimiento demográfico tras la Guerra de los Siete Años, que, ya en las últimas décadas del siglo XVIII, tiene como consecuencia una generación de jóvenes, más o menos instruidos formalmente, cuyos futuros no estaban para nada asegurados en el territorio germano y que acabarán siendo, por no tener lugar que reclamar en sus naciones, incipientes inconformistas literarios, críticos culturales (Whaley, 2003: 50); las discusiones estético-filosóficas del siglo XVIII, desde nociones ilustradas hasta la particular recepción de posiblemente el padre de la Kulturkritik, Rousseau, quien también estaba interesado, si se quiere, en el tiempo justo de la formación, como advertimos en la cronología de su Emilio; ${ }^{16}$ la ambivalente postura frente a la Revolución Francesa, de promesa inicial a la percepción de la revolución como fracaso o traición, y las consecuencias en la concepción del devenir de la historia a partir de este acontecimiento; $\mathrm{y}$, ligado al primer elemento, la creciente importancia de la figura del joven autor y de la juventud como ideal, casi siempre, hermanado al concepto de lo nuevo estético, algo que vemos con claridad, primero, en el Sturm und Drang y, luego, en el Romanticismo de Jena. Sin duda, el grado de influencia específica de cada uno de estos factores en la construcción temporal de la novela de formación constituye un tema a investigar en sí mismo.

Pero, lejos de establecer una línea de determinación unívoca entre estos fenómenos y la literatura de esa época, lo que se debe reconocer es la relevancia del tiempo en el contexto en el que se inserta y en el que debate la novela goetheana en sus cuantiosos diálogos. Luego, por el rol modélico en el que es ubicada la novela, lega al Bildungsroman alemán, aunque sea como modelo a revisar críticamente, estas preocupaciones sobre el tiempo, tanto en el plano de la narración (cómo narrar, con qué tiempo narrar, qué focalizar, qué contraer, etc.), como en el formativo (en qué momento el sujeto se puede formar, por ejemplo). En la medida en que reflexionen sobre la Bildung, persiste, con variaciones, el interés por el tiempo, como problema de representación; como instrumento para configurar (y medir) la identidad de un sujeto a través de mecanismos de olvido (y, por la 
escritura, de memoria); o como modo de pensar las posibilidades de formación en relación a períodos temporales (lo que supone focalizar la juventud en tanto "tiempo de la formación"). ${ }^{17}$

El Bildungsroman es así un subgénero -ampliando la autoconciencia epistemológica que Swales lee en el subgénero (1991: 66)- sumamente auto-consciente de la representación del tiempo. De Goethe a Mann, los narradores elaboran, una y otra vez, con sus específicas variaciones en las tres variables que analizamos, la relación entre tiempo y formación. Deberíamos, a modo de conclusión, pues, extender a todos los Bildungsromane -o, cuando menos, a los escritos en alemánla afirmación que el narrador aplica a La montaña mágica (Mann, 2002), en tanto que todos serían, en mayor o menor medida, novelas temporales, novelas del y sobre el tiempo. 


\section{Q Bibliografía}

" Adorno, T. W. (2003). Sobre el clasicismo en la Ifigenia de Goethe. En Notas sobre literatura. Obra Completa 11, pp. 475-494. Trad. de A. B. Muñoz. Madrid: Akal.

" Auerbach, E. (1996). Mimesis: La representación de la realidad en la literatura occidental. Trad. de J. Villanueva y E. İmaz. México: Fondo de Cultura Económica.

"Bajtín, M. M. (2013). La novela de educación y su importancia en la historia del realismo. En Estética de la creación verbal, pp. 197-244. Trad. de T. Bubnova. Buenos Aires: Siglo XXI.

" Finkielkraut, A. y Sloterdijk, P. (2008). Retornos. En Los latidos del mundo. Diálogo, pp. 9-26. Trad. de H. Cardoso Franco. Buenos Aires: Amorrortu.

" Gillis, J. R. (1981). Youth and History: Tradition and Change in European Age Relations, 1770-Present. Londres: Academic Press.

" Goethe, J. W. (2019). Los años de aprendizaje de Wilhelm Meister. Trad. de M. Salmerón. Madrid: Cátedra.

" Goethe, J. W. (2017). Los años itinerantes de Wilhelm Meister. Trad. de M. Salmerón. Madrid: Cátedra.

"Goethe, J. W. (1991). Diderots Versuch über die Malerei. En Sämtliche Werke nach Epochen seines Schaffens. Bd. 7: Leben des Benvenuto Cellini. Diderots Versuch über die Malerei. Rameaus Neffe, pp. 517-565. Múnich: Hanser.

" Hamburger, K. (1994). Die Logik der Dichtung. Stuttgart: Klett Cotta.

" Jacobs, J. (1981). Wilhelm Meister und Seine Brüder. Untersuchungen zum deutschen Bildungsroman. Múnich: Wilhelm Fink.

" Kant, I. (2013). ¿Qué es la llustración? Ed. Roberto R. Aramayo. Madrid: Alianza.

"Koval, M. I. (2018). Vocación y renuncia: La novela de formación alemana entre la Ilustración y la Primera Guerra Mundial. Buenos Aires: Facultad de Filosofía y Letras Universidad de Buenos Aires.

"Lukács, G. (2002). La novela de educación. En Teoría de la novela. Ensayo histórico filosófico sobre las formas de la gran literatura épica, pp. 116-126. Trad. M. Sacristán. Madrid: Biblioteca Nacional.

" Mann, T. (2002). La montaña mágica. Trad. de M. Verdaguer. Barcelona: Edhasa.

" Mann, T. (1960). Gesammelte Werke, XI. Fráncfort del Meno: S. Fischer.

"Martínez, M. y Scheffel, M. (2011). Introducción a la narratología. Hacia un modelo analítico-descriptivo de la narración ficcional. Trad. de M. Koval. Buenos Aires: Las Cuarenta.

" Mendelssohn, M. (1784). Ueber die Frage: was heißt aufklären?. En Berlinische Monatsschrift, vol. 4, pp. 193-200. Universität Bielefeld. <http://ds.ub.uni-bielefeld. de/viewer/resolver?urn=urn:nbn:de:0070-disa-2239816_004_2777> [Consulta: 14 de febrero de 2020]

" Miles, D. H. (1974). The Picaro's Journey to the Confessional: The Chaning Image of the Hero in the German Bildungsroman. PMLA, vol. 85, núm. 5, pp. 980-992. <https://doi.org/10,2307/461371> [Consulta: 14 de febrero de 2020]

"Moretti, F. (1987). The Bildungsroman as Symbolic Form. En The Way of the World: 
The Bildungsroman in European Culture, pp. 3-14. Norfolk: Verso.

" Moretti, F. (1985). The Comfort of Civilization. Representations, núm. 12, pp. 115-139.

" Müller, G. (2014). Über das Zeitgerüst des Erzählens. Erich Rothacker zum 60 Geburtstag. En Morphologische Poetik: Gesammelte Aufsätze, pp. 388-418. Ed. Elena Müller. Berlin: Walter de Gruyter.

"Novalis. (1978). Werke in drei Bänden. Múnich: Hanser.

"Ricœur, P. (2008). Tiempo y narración Il: Configuración del tiempo en el relato de ficción. Trad. de A. Neira. México: Siglo XXI.

" Roseman, M. (ed.). (2003). Generations in Conflict: Youth revolt and generation formation in Germany 1770-1968. Cambridge: Cambridge University Press.

"Sammons, J. L. (1991). The Bildungsroman for Nonspecialists: An Attempt at a Clarification. En Hardin, J. N. (ed.). Reflection and Action: Essays On the Bildungsroman, pp. 26-45. Columbia: University of South Carolina Press.

"Steinby, L. (2017). Temporality, Subjectivity and the Representation of Characters in the Eighteenth-Century Novel from Defoe's Moll Flanders to Goethe's Wilhelm Meisters Lehrjahre. En Steinby, L. y Mäkikalli, A. (eds.). Narrative Concepts in the Study of Eighteenth-Century Literature, pp. 135-160. Amsterdam: Amsterdam University Press. <https://doi.org/10,2307/j.cttlwnor6q> [Consulta: 14 de febrero de 2020]

"Steinecke, H. (1991). The Novel and the Individual: The Significance of Goethe's Wilhelm Meister in the Debate about the Bildungsroman. En Hardin, J. N. (ed.). Reflection and Action: Essays On the Bildungsroman, pp. 69-96. Columbia: University of South Carolina Press.

"Vedda, M. (2014). Alegorías de la Modernidad: el Wilhelm Meister, desde la novela teatral a la novela-archivo. En Leer a Goethe, pp. 141-184. Buenos Aires: Quadrata,

"Watt, I. (1957). Realism and the novel form. En The Rise of the Novel: Studies in Defoe, Richardson, and Fielding, pp. 9-34. Londres: Chatto \& Windus.

"Whaley, J. (2003). The ideal of youth in late eighteenth-century Germany. En Roseman, M. (ed.). Generations in Conflict: Youth revolt and generation formation in Germany 1770-1968, pp. 47-68. Cambridge: Cambridge University Press. 